\title{
Work softening and annealing hardening of deformed nanocrystalline nickel
}

\author{
X. Y. Zhang, ${ }^{1,2, a)}$ Q. Liu, ${ }^{1, b)}$ X. L. Wu, ${ }^{3}$ and A. W. Zhu ${ }^{2}$ \\ ${ }^{1}$ School of Materials Science and Engineering, Chongqing University, Chongqing 400030, \\ People's Republic of China \\ ${ }^{2}$ Physical Science and Engineering Technology School, Guangxi University, Nanning 530004, \\ People's Republic of China \\ ${ }^{3}$ State Key Laboratory of Nonlinear Mechanics, Institute of Mechanics, Chinese Academy of Sciences, \\ Beijing 100190, People's Republic of China
}

(Received 7 November 2008; accepted 10 December 2008; published online 31 December 2008)

\begin{abstract}
We reported that work softening takes place during room-temperature rolling of nanocrystalline $\mathrm{Ni}$ at an equivalent strain of around 0.30 . The work softening corresponds to a strain-induced phase transformation from a face-centered cubic (fcc) to a body-centered cubic (bcc) lattice. The hardness decreases with increasing volume fraction of the bcc phase. When the deformed samples are annealed at $423 \mathrm{~K}$, a hardening of the samples takes place. This hardening by annealing can be attributed to a variety of factors including the recovery transformation from the bcc to the fcc phase, grain boundary relaxation, and retardation of dislocation gliding by microtwins. (C) 2008 American Institute of Physics. [DOI: 10.1063/1.3062849]
\end{abstract}

The deformation mechanisms of nanocrystalline (nc) materials are very different from those of coarse grain materials. ${ }^{1-7}$ It has been found that in face-centered cubic (fcc) nc Ni, mechanisms such as deformation twinning, formation of extended and full dislocations from the grain boundaries (GBs), GB sliding, and grain rotation can contribute to plasticity. ${ }^{6,8-16}$ Recently, we have reported that for $\mathrm{nc} \mathrm{Ni}$, a phase transformation from fcc $(\gamma)$ to body-centered cubic (bcc, $\alpha$ ) can provide another deformation mechanism to realize plastic deformation to relatively large plastic strain during room-temperature rolling, ${ }^{17}$ i.e., a far-fromequilibrium microstructure such as nc $\mathrm{Ni}$ can accommodate plastic strain via a change in lattice structure upon mechanical loading. In this letter we report that such a strain-induced phase transformation from $\gamma$ to $\alpha$ leads to work softening during cold-rolling and that subsequent annealing at $423 \mathrm{~K}$ results in hardening.

Fully dense, electrodeposited nc Ni sheets $(99.8 \%$ purity) were procured from Goodfellow, Inc. The as-received sheets were $200 \mu \mathrm{m}$ thick with an average grain size of about $20 \mathrm{~nm}$. Samples of the $\mathrm{nc} \mathrm{Ni}$ of $10 \times 10 \mathrm{~mm}^{2}$ in size were rolled at room temperature to various von Mises equivalent strains $\varepsilon_{\mathrm{VM}}$ calculated as $\varepsilon_{\mathrm{VM}}=|2 / \sqrt{3} \ln (1+\delta)|$, where $\delta$ is the rolling reduction. The microstructure of the samples was examined using $\mathrm{x}$-ray diffraction (XRD) (Riguta D/max-RC) and high-resolution transmission electron microscopy (JEM 2010F operated at $200 \mathrm{kV}$ ). Microhardness measurements were taken using a load of $0.196 \mathrm{~N}$ (HVS-1000). The reported hardness values are each averaged from three indents. The hardness provides a measure of the resistance to deformation by surface indentation or by abrasion and is a combined property of elasticity, plasticity, strength, and toughness. However, the hardness value is determined by the resistance both to initial and continuous plastic deformation. In general, a high hardness corresponds

\footnotetext{
a) Electronic mail: kehen888@163.com.

${ }^{b)}$ Electronic mail: qingliu@ @ cqu.edu.cn.
}

to a high tensile strength, although there is not an exact relationship between the two values. Considering the small dimensions of the deformed samples, in the experiment we use the sample hardness to investigate changes in the mechanical properties.

The experimental result shows that once a deformation strain of $\varepsilon_{\mathrm{VM}}=0.32$ is reached, the $\alpha$ phase starts to appear by a strain-induced martensitic transformation. ${ }^{17}$ Figure $1(\mathrm{a})$
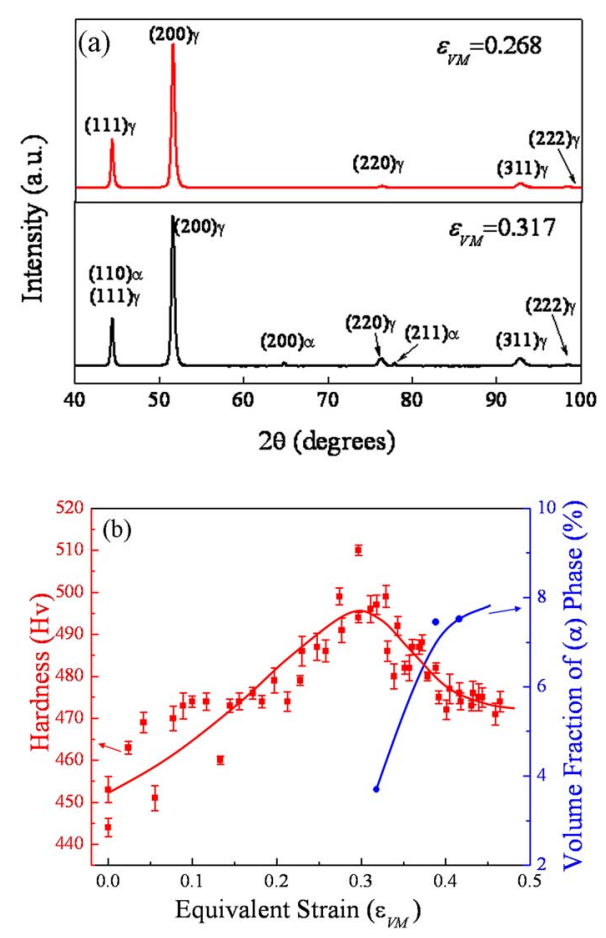

FIG. 1. (Color online) (a) XRD profiles of samples rolled to equivalent strains of 0.268 and 0.32 . bcc diffraction peaks are not detected at $\varepsilon_{\mathrm{VM}}$ $=0.268$ and are detected at $\varepsilon_{\mathrm{VM}}=0.32$. (b) Work softening takes place as $\varepsilon_{\mathrm{VM}}$ exceeds at about 0.30 . At the same time transformation to the $\alpha$ phase occurs. After a strain of about 0.30, the decrease in hardness (red line) corresponds to the increase in volume fraction of the $\alpha$ phase (blue line). 

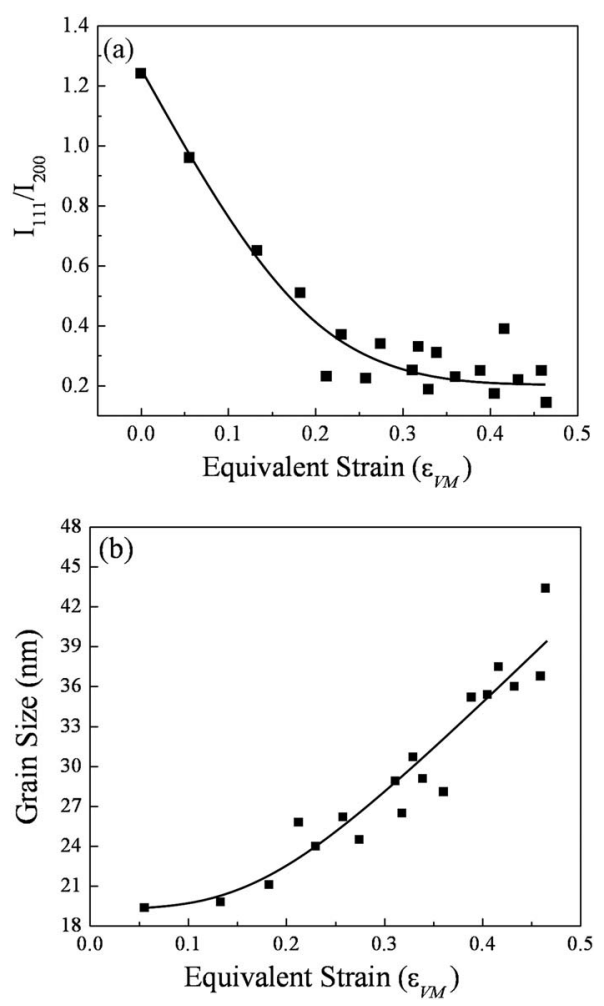

FIG. 2. The evolution of texture (a) and the change in grain size (b) with increasing plastic strain.

shows an example of XRD results for nc Ni rolled to $\varepsilon_{\mathrm{VM}}$ $=0.32$. Figure $1(\mathrm{~b})$ displays the change in hardness for samples rolled to various strains. It can be seen that before the strain of about 0.3 , the hardness increases with increasing plastic strain. Interestingly, after the strain of about 0.3 the hardness decreases with increasing plastic strain, indicating that work softening takes place. This work softening corresponds to the phase transformation from $\gamma$ to $\alpha$. The hardness decreases with increasing volume fraction of the $\alpha$ phase $\left(f_{\alpha}\right)$. This behavior is different from displacive martensitic transformations as seen in polycrystalline alloys, which are typically characterized by solid solution hardening. ${ }^{18}$

The evolution of the texture and grain size with increasing plastic strain was also investigated. Figure 2(a) shows change in the $(111) /(200)$ diffraction intensity ratio $\left(I_{111} / I_{200}\right)$ with increasing strain. A preferred (200) orientation is seen in the original undeformed sample $\left(I_{111} / I_{200}=1.24\right.$, for nontextured polycrystalline $\left.\mathrm{Ni} I_{111} / I_{200}=2.05\right) .{ }^{99}$ It can be seen that in the early stages of cold rolling, the (200) preferred orientation strengthens [Fig. 2(a)], the grain size increases [Fig. 2(b)], and work hardening takes place [Fig. 1(b)] with increasing strain. These results therefore imply that in the early stages of deformation, plastic flow is dominated by dislocation activity and grain rotation. ${ }^{20}$ Figure 2(a) also shows that beyond a strain of $\varepsilon_{\mathrm{VM}}=0.2$, the rate of strengthening of preferred (200) orientation begins to decrease, indicating that the dislocation activity and grain rotation become difficult. It has been suggested that at large plastic strains deformation, twinning and formation of extended and full dislocations from GBs play an important role. ${ }^{14,15}$ Once $\varepsilon_{\mathrm{VM}}$ exceeds $\sim 0.30$, the preferred orientation changes little [Fig. $2(\mathrm{a})]$ and work softening takes place [Fig. 1(b)]. At the same time, a phase transformation from $\gamma$ to $\alpha$ starts to accommodate the plastic deformation. ${ }^{17}$ As shown in Fig. 1(b), the

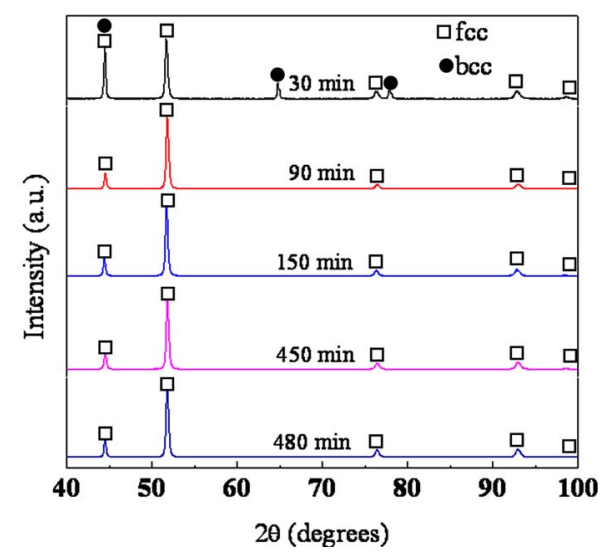

FIG. 3. (Color online) XRD profiles of annealed samples. The deformed sample $\left(\varepsilon_{\mathrm{VM}}=0.497\right)$ was annealed at $423 \mathrm{~K}$ for different times. Diffraction peaks of the $\alpha$ phase can still be detected for sample annealed for $30 \mathrm{~min}$. For annealing times of more than $90 \mathrm{~min}$, the $\alpha$ phase is not detected.

sample hardness decreases with increasing volume fraction of the $\alpha$ phase, indicating an inversely proportional relationship between the sample hardness and the volume fraction of the $\alpha$ phase. These observations imply that the $\gamma \rightarrow \alpha$ transformation relaxes the high inhomogeneous internal stresses that results from elastic anisotropy and plastic incompatibilities in different grains or in different parts of each grain, ${ }^{14,21}$ leading to a decrease in the hardness. For example, as $\varepsilon_{\mathrm{VM}}$ increases from 0.32 to 0.42 , the volume fraction of the $\alpha$ phase increases from $3.5 \%$ to $7.5 \%$ and the hardness decreases from $490 \mathrm{Hv}$ to $470 \mathrm{Hv}$. The transition in behavior occurs at an equivalent strain of about 0.30 . At higher plastic strains, work hardening changes into work softening, indicating that the transformation from one lattice into another begins to accommodate plastic strain upon continued mechanical loading. It can also be seen from Fig. 2(b) that the grain size increases during cold-rolling. It is believed that the variation in the grain size also has an influence on the mechanical properties. However, as shown in Fig. 2(b), the rate of grain growth during the work hardening region is the same as that during the work softening region. It is not certain to what extent the hardness drops due to the increase in grain size can be accounted by the Hall-Petch formula, and this should be considered carefully in further studies.

Deformed samples in which $\alpha$ phase was detected by XRD were annealed at $423 \mathrm{~K}$ for different times, after which the annealed samples were examined again by XRD. The results are shown in Fig. 3. It can be seen that after $30 \mathrm{~min}$ annealing, diffraction peaks of the $\alpha$ phase can still be detected. For samples annealed for $90 \mathrm{~min}$ or longer, the $\alpha$ phase diffraction peaks disappear. This phenomenon indicates that a recovery transformation from the $\alpha$ to the $\gamma$ phase takes place during annealing. Figure 4 shows the change in sample hardness with increasing annealing time for samples deformed to equivalent strains $\left(\varepsilon_{\mathrm{VM}}\right)$ of 0.493 (black) and 0.12 (red). It can be seen that hardening takes place during the annealing. The phenomenon of hardening by annealing has also been reported for nc Ni (Ref. 22) and nc $\mathrm{Al}^{23}$ However, the circumstances in these reports are different from those in our experiment. For $\mathrm{nc} \mathrm{Ni},{ }^{22}$ hardening by annealing was seen only in an as-electrodeposited sample (grain size of about $29 \mathrm{~nm}$ ) and not in a deformed sample. When the sample was annealed at $423 \mathrm{~K}$, the strength in- 


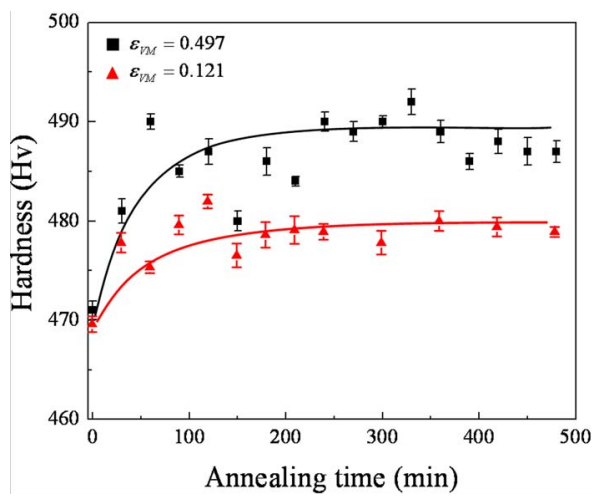

FIG. 4. (Color online) Change in sample hardness with increasing annealing time for samples deformed to equivalent strains $\left(\varepsilon_{\mathrm{VM}}\right)$ of 0.493 (black) and 0.12 (red).

creased without obvious coarsening of the grain size. This result was attributed to a decrease in GB stresses and the consequent difficulty of the emission of dislocations from $\mathrm{GBs}^{22}$ For the deformed $\mathrm{nc} \mathrm{Al},{ }^{23}$ annealing resulted in the disappearance of dislocation boundaries and a decrease in the dislocation density. This may lead to an increase in the stress required to activate new dislocation sources during straining. The nc Al samples were prepared by accumulated rolling bonding (ARB), and the grain size was characterized by the average spacing of the lamellar boundaries as typically seen in the microstructure of ARB metals. The average lamellar boundary spacing was given as $180-600 \mathrm{~nm}$. Consequently the behavior may not represent that for nc metals of a smaller grain size (for example, $20 \mathrm{~nm}$ ). In our deformed samples, we also observed deformation twinning, stacking faults, and dislocations. It has been reported that in low temperature annealing, internal stresses may be restored partially by GB atom readjustments. Such GB relaxation will result in increased difficulty for GB emission of dislocations. Additionally, microtwin can also retard glide of dislocations. ${ }^{24}$ The misfit atoms in a twin boundary provide a driving force for twin growth during annealing, leading to an increased difficulty for gliding of dislocations in the grain interiors. ${ }^{25}$ The hardening by annealing in Fig. 4 can be attributed in part to these factors (see the red line for $\varepsilon_{\mathrm{VM}}=0.12$ ). Also, from Fig. 4 it can be seen that for the $\varepsilon_{\mathrm{VM}}=0.493$ sample, the hardness increases quickly from $473 \mathrm{Hv}$ to about $487 \mathrm{Hv}$ in the early stages of the annealing (black line). This increase corresponds to a reversal of the $\alpha$ to $\gamma$ transformation (see Fig. 3). For annealing times exceeding $90 \mathrm{~min}$, the hardness changes little. Correspondingly, diffraction peaks of the $\alpha$ phase cannot be detected by XRD. Therefore, we believe that for the $\varepsilon_{\mathrm{VM}}=0.493$ sample, the hardening by annealing may be also partially attributed to the reversed transformation from the $\alpha$ to the $\gamma$ phase in addition to GB relaxation and retardation of dislocation gliding by microtwins.

In summary, in this letter we have reported that a work softening takes place during room-temperature rolling of nc Ni. The work softening is accompanied by a transformation from $\gamma$ to $\alpha$ structure. The sample hardness decreases with increasing volume fraction of the $\alpha$ phase. When the coldrolled samples are annealed at a temperature of $423 \mathrm{~K}$, the hardness increases with increasing annealing time. This hardening can be attributed to a number of factors including a recovery transformation from the $\alpha$ to the $\gamma$ phase, GB relaxation, and blockage of dislocation glide by microtwins.

This work was supported by NSFC under Grant Nos. 50571110, 10721202, and 50461001.

${ }^{1}$ V. Yamakov, D. Wolf, S. R. Phillpot, A. Mukherjee, and H. Gleiter, Nature Mater. 1, 45 (2002)

${ }^{2}$ V. Yamakov, D. Wolf, S. R. Phillpot, A. Mukherjee, and H. Gleiter, Nature Mater. 3, 43 (2004)

${ }^{3}$ H. Swygenhoven, P. M. Derlet, and A. Froseth, Nature Mater. 3, 399 (2004).

${ }^{4}$ M. W. Chen, E. Ma, K. J. Hemker, H. W. Sheng, Y. M. Wang, and X. M. Cheng, Science 300, 1275 (2003).

${ }^{5}$ R. J. Asaro and S. Suresh, Acta Mater. 53, 3369 (2005).

${ }^{6}$ M. A. Meyers, A. Mishra, and D. J. Benson, Prog. Mater. Sci. 51, 427 (2006).

${ }^{7}$ H. Gleiter, Acta Mater. 48, 1 (2000).

${ }^{8}$ Y. T. Zhu, X. Z. Liao, S. G. Srinivasan, Y. H. Zhao, M. I. Baskes, F. Zhou, and E. J. Lavernia, Appl. Phys. Lett. 85, 5049 (2004).

${ }^{9}$ X. Z. Liao, F. Zhou, E. J. Lavernia, S. G. Srinivasan, I. Baskes, D. W. He, and Y. T. Zhu, Appl. Phys. Lett. 83, 632 (2003).

${ }^{10}$ X. Z. Liao, F. Zhou, E. J. Lavernia, D. W. He, and Y. T. Zhu, Appl. Phys. Lett. 83, 5062 (2003).

${ }^{11}$ J. Weissmuller and J. Markmann, Adv. Eng. Mater. 7, 202 (2005).

${ }^{12}$ J. Wang and H. C. Huang, Appl. Phys. Lett. 85, 5983 (2004).

${ }^{13}$ Z. W. Shan, E. A. Stach, J. M. K. Wiezorek, J. A. Knapp, D. M. Follstaedt, and S. X. Mao, Science 305, 654 (2004).

${ }^{14}$ X. L. Wu and E. Ma, Appl. Phys. Lett. 88, 061905 (2006).

${ }^{15}$ X. L. Wu and E. Ma, Appl. Phys. Lett. 88, 231911 (2006).

${ }^{16}$ X. Wu, Y. T. Zhu, M. W. Chen, and E. Ma, Scr. Mater. 54, 1685 (2006)

${ }^{17}$ X. Y. Zhang, X. L. Wu, Q. Liu, R. L. Zuo, A. W. Zhu, P. Jiang, and Q. M. Wei, Appl. Phys. Lett. 93, 031901 (2008).

${ }^{18} \mathrm{~J}$. W. Christian and D. V. Edmonds, in Phase Transformations in Ferrous Alloys, edited by A. R. Marder and J. L. Goldstein (American Society for Metals, Metals Park, OH, 1984), pp. 293-325.

${ }^{19}$ Z. Budrovic, H. Van Swygenhoven, P. M. Derlet, S. Van Petegem, and B. Schmitt, Science 304, 273 (2004).

${ }^{20}$ Q. Liu, X. Huang, D. J. Lloyd, and N. Hansen, Acta Mater. 50, 3789 (2002).

${ }^{21}$ J. G. Wright and J. Goddard, Philos. Mag. 11, 485 (1965).

${ }^{22}$ Y. M. Wang, S. Cheng, Q. M. Wei, E. Ma, T. G. Nieh, and A. Hamza, Scr. Mater. 51, 1023 (2004).

${ }^{23}$ X. Huang, N. Hansen, and N. Tsuji, Science 312, 249 (2006).

${ }^{24}$ L. Lu, Y. F. Shen, X. H. Chen, L. H. Qian, and K. Lu, Science 304, 422 (2004).

${ }^{25}$ N. Jia, Y. D. Wang, S. D. Wu, W. Z. Han, Y. N. Wang, J. N. Deng, and P. K. Liaw, Scr. Mater. 54, 1247 (2006). 